\title{
QUANTITATIVE STRUCTURE-ACTIVITY RELATIONSHIP (QSAR) OF N-BENZOYL-N'-PHENYLTHIOUREA COMPOUND AND DERIVATIVES IN MCF-7 CANCER CELLS
}

\author{
D. Kesuma ${ }^{1}$, H. Santosa ${ }^{1}$, A.L. Nasyanka ${ }^{2, 凶}$, and Ruswanto ${ }^{3}$ \\ ${ }^{1}$ Department of Medicinal Chemistry, Faculty of Pharmacy, University of Surabaya, Surabaya, \\ (East Java) Indonesia, 60293. \\ ${ }^{2}$ Departement of Pharmacy, Faculty of Health, University of Muhammadiyah Gresik, Gresik, \\ (East Java) Indonesia, 61111. \\ ${ }^{3}$ Departement of Pharmacy, Sekolah Tinggi Ilmu Kesehatan Bakti Tunas Husada, Tasikmalaya-, \\ (West Java) Indonesia, 6115. \\ ${ }^{\square}$ Corresponding Author: anindilupita@umg.ac.id
}

\begin{abstract}
Thiourea derivatives are one of the promising anticancer groups to be developed further. These derivatives act as an EGFR inhibitor by inhibiting Tyrosine Kinase Receptors (RTKs) in the intracellular region. In this study, a compound of thiourea derivative, namely N-benzoyl-N'-phenylthiourea (BFTU) compound, was synthesized from Nphenylthiourea through an acylation reaction with benzoyl chloride. In addition, it also synthesized 4 BFTU derivatives compounds, namely: 2-Cl-BFTU; 3-Cl-BFTU; 4-Cl-BFTU; 2,4-2Cl-BFTU. The synthesis of 5 compounds was carried out through a single-step reaction and the structure of the synthesis result was confirmed using IR, ${ }^{1} \mathrm{H}$ NMR, ${ }^{13} \mathrm{C}-\mathrm{NMR}$, HRMS. To determine the $\mathrm{IC}_{50}$ cytotoxic activity of BFTU compound and its 4 derivatives, in vitro activity test was conducted against breast cancer cells MCF-7 and their selectivity in Vero normal cells. BFTU compound and its 4 derivatives showed higher cytotoxic activity on MCF-7 cells than Hydroxyurea (HU) compound and 4 BFTU derivatives showed higher cytotoxic activity than Erlotinib. BFTU compound and its 4 derivatives are toxic to MCF-7 cells but selective or not toxic to Vero normal cells. The best QSAR equation, namely: $\log 1 / \mathrm{IC}_{50}=$ $0.354 \pi+0.064(\mathrm{n}=5 ; \mathrm{r}=0.922 ; \mathrm{SE}=0.864 ; \mathrm{F}=16.953 ; \mathrm{Sig}=0.026)$. The best QSAR equation obtained can be used to design other BFTU derivative compounds, which have better anticancer activity.

Keywords: N-benzoyl-N'-phenylthiourea, Synthesis, Cytotoxic Activity, MCF-7 cells, QSAR

RASĀYAN J. Chem., Vol. 14, No.4, 2021
\end{abstract}

\section{INTRODUCTION}

Drug design is an effort to develop existing drugs with acknowledged molecular structure and biological activity. It is conducted based on systematic and rational reasoning by minimizing trials and errors. ${ }^{1} \mathrm{~A}$ rational drug design is a design to discover new drugs logically, which then can be explained theoretically using the Quantitative Structure-Activity Relationship (QSAR) approach. The advancement of technology nowadays has resulted in more rapid conduct of QSAR approach. Moreover, technology also enables a more specific drug design using molecular modeling techniques with computer assistance known as Computer-Aided Drug Design (CADD). ${ }^{2}$

It is highly crucial to develop new cancer drugs considering that the disease has become a significant cause of death worldwide following cardiovascular disease. And the primary cause of death amongst Indonesian women is breast cancer. ${ }^{3}$ The high prevalence of cancer requires precautionary measures as well as swift, appropriate treatment. On the other hand, medicines that have long been used gradually become less effective. Moreover, cancer cells tend to become resistant to current cancer drugs nowadays. ${ }^{4}$

Thiourea is a compound containing sulfur and nitrogen atoms with a chemical structure similar to urea compounds that have been used as anticancer, including Hydroxyurea, Nitrosourea, 5-Fluorouracil and Sorafenib. ${ }^{5,6}$ Thiourea derivatives are one of the high potential anticancer groups to develop further. This derivative acts as an EGFR (Epidermal Growth Factor Receptor) inhibitor by inhibiting Tyrosine Kinase Receptors (RTKs) in the intracellular region. Some researchers have synthesized thiourea derivative Rasayan J. Chem., 14(4), 2698-2704(2021)

http://dx.doi.org/10.31788/RJC.2021.1446357

This work is licensed under a CC BY 4.0 license. 


\section{RASĀYAN $J$. Chem.}

Vol. 14 | No. 4 |2698-2704| October- December | 2021

compounds and tested their activity on EGFR inhibition. This proves that some thiourea derivatives bind well to EGFR, which can result in inhibition of the tumor cell proliferation process. ${ }^{7,8}$

This research synthesized several new compounds of thiourea derivatives, namely N-benzoyl-N'phenylthiourea (BFTU), synthesized from N-phenylthiourea through an acylation reaction with benzoyl chloride. In addition, it also synthesized 4 BFTU derivatives compounds (except 3-Cl as a functional group that has been synthesized before). The synthesis of these 5 compounds was carried out through a singlestep reaction and continued with testing the cytotoxic activity in vitro on MCF-7 cancer cells and Vero normal cells and Quantitative Structure-Activity Relationship (QSAR) analysis. ${ }^{9-10}$ The BFTU lead compound was modified by incorporating clusters on the aromatic ring based on the Topliss approach method into several derivative compounds. Modifications were arranged by considering the lipophilic and electronic factors of various substituents and were designed in a way that optimal substituents could be found as efficiently as possible. The reagents used were phenylthiourea and benzoyl chloride derivatives with various substituents, namely: $2-\mathrm{Cl} ; 3-\mathrm{Cl} ; 4-\mathrm{Cl} ; 2,4-2 \mathrm{Cl}$. The modification was conducted on the basis of theory stating that the biological activity of a compound is affected by the psycho-chemical properties categorized into lypophillic, electronic dan steric properties. The lipophilic property affects the capability of a compound to penetrate biological membrane while electronic property influences mainly on drugreceptor interaction process as well as on biological membrane penetration. As for steric property, it determines the compatibility of molecular compound interaction with the receptors within the cells. ${ }^{11,12}$ BFTU compound and its 4 derivatives were synthesized from $\mathrm{N}$-phenylthiourea through an acylation reaction with benzoyl chloride and 4 derivatives. ${ }^{13}$ Confirmation of the structure of the BFTU compound and its 4 derivatives was carried out with analytical instruments, namely: IR spectrophotometry, ${ }^{1} \mathrm{H}-\mathrm{NMR}$ and ${ }^{13} \mathrm{C}$-NMR spectrometry and MS spectrometry. ${ }^{14,15}$ Cytotoxicity test aims to measure the cytotoxic ability of test compounds.. against cell growth. In this study, cytotoxic activity tests were carried out using the MTT assay method. IC $_{50}$ determination of the cytotoxic activity of BFTU compound and its 4 derivatives were tested for in vitro activity against human breast cancer cells, namely MCF-7 cancer cells and vero normal cells. MCF-7 cancer cells are the most widely used human breast cancer cell culture in research and EGFR overexpression. ${ }^{16}$ As a comparison, anticancer compounds of hydroxyurea were used. They have a similar pharmacophore structure with BFTU compounds. Also, erlotinib compounds are known to have cancer cell pathway inhibition activity of EGFR pathways. A lower $\mathrm{IC}_{50}$ value means a more potent compound. ${ }^{18-19}$

The quantitative relationship between BFTU compound and its 4 derivatives with cytotoxic activity in vitro is expressed through linear or non-linear regression equations as a dependent parameter is the cytotoxic activity $\left(\log 1 / \mathrm{IC}_{50}\right)$ of BFTU compounds and their derivatives against MCF-7 cells. The independent parameters used in this relationship are the parameters of physicochemical properties, including lipophilic parameters: $\mathrm{Clog} \mathrm{P}$ and $\pi$, sterics: CMR and Es, and electronic parameters: Etot and $\sigma$. The best QSAR equation obtained can be used to design other BFTU derivative compounds which will possess better anticancer activity. ${ }^{20-22}$

\section{Material and Methods}

\section{EXPERIMENTAL}

Materials for this synthesis include N-phenylthiourea; benzoylchloride; 2-chlorobenzoyl chloride; 3chlorobenzoyl chloride; 4-chlorobenzoyl chloride; 2,4-dichlorobenzoyl chloride of Sigma Aldrich. Tetrahydrofuran (THF), Trietylamin (TEA), aceton, ethyl acetate, n-heksana, chloroform, ethanol and natrium bicarbonate $\left(\mathrm{NaHCO}_{3}\right)$ p.a of E. Merck; Kieselgel $60 \mathrm{~F}_{254}$ of E. Merck.

The instruments used for synthesis and structure confirmation are : test tube for synthesis, Corning Hot Plate P351, Fisher-John Electrothermal Mel-Temp, Jasco FT-IR 5300, Spectrophotometry ${ }^{1} \mathrm{H}-\mathrm{NMR}$ and ${ }^{13} \mathrm{C}-\mathrm{NMR}$ Agilent (400 and $100 \mathrm{MHz}$ ), Spectrometry ${ }^{1} \mathrm{H}-\mathrm{NMR}$ and ${ }^{13} \mathrm{C}-\mathrm{NMR}$ Agilent $500 \mathrm{MHz}$ with DD2 console system operating on $500 \mathrm{MHz}\left({ }^{1} \mathrm{H}\right)$ and $125 \mathrm{MHz}\left({ }^{13} \mathrm{C}\right)$ frequency, Mass Spectrometry Waters LCT Premier XE Detector TOF acethon solvent $+0,1 \%$ formatted acid in acetonitryl - water $(1: 1)$.

\section{Materials for Activity Test}

5 compounds resulted from the synthesis, hydroxyurea, erlotinib, MCF-7 cell culture and Vero cell. DMEM and M199 culture media, phosphate-buffered saline (PBS), FBS (Fetal Bovine Serum), Tripsin, Penicillin- 
Streptomicin, Fungizon, DMSO, $0.5 \mathrm{mg} / \mathrm{mL}$ of MTT (3-(4,5-dimetiltiazol-2-il)-2,5-difeniltetrazolium bromida) , SDS $10 \%$ in $\mathrm{HCl} 0,1 \mathrm{~N}$.

\section{Instruments for Cytotoxic Test}

$\mathrm{CO}_{2}$ Incubator (Hera cell), LAF (Gelman Sciences), micropippets 20, 200, $1000 \mu \mathrm{L}$ with a blue and yellow tip, culture tube, vortex, microplate 96 well, Conical tube, Inverted Microscope (Zeiss 451235), hemocytometer, cell counter, ELISA-reader (Bio-Rad).

\section{Synthesis Method}

The synthesis method uses schotten baumann reaction with one-step procedure. First, on a round-bottom flask are $0,0080 \mathrm{~mol}$ of $\mathrm{N}$-phenylthiourea mingled with tetrahydrofuran and 0,0075 mol of TEA. On ice bath, $0.0075 \mathrm{~mol}$ of benzoyl chloride solution and its derivatives were gradually added into tetrahydrofuran through dropping funnel while operated on magnetic stirrers for 30 minutes. When finished, the mixture was then refluxed on a water bath and was analyzed their purity using thin layer chromatography per one hour. The reaction was halted when spots merged into one single spot or when spots of benzoyl chloride and its derivatives have disappeared. After that termination, tetrahydrofuran was evaporated using rotavapor. The concentrated outcome of the reaction was rinsed with saturated $\mathrm{NaHCO}_{3}$. Filtered with Buchner funnel, the result was rinsed with water. Then, using hot ethanol, recrystallization was performed. The formed crystal, whose mass was previously recorded, was removed to petridish and then dried in a stable oven temperature of $50^{\circ} \mathrm{C}$. The identification and confirmation of synthesized compounds were conducted on the basis of identification result using IR, ${ }^{1} \mathrm{H}-\mathrm{NMR},{ }^{13} \mathrm{C}-\mathrm{NMR}$, HRMS.

\section{Cytotoxic Test Method}

A plate containing the prepared cells was removed from the incubator, media was discarded and the remaining liquid was drained. By adding $100 \mu \mathrm{L}$ of PBS into cell-containing wells, the cells were cleansed and PBS was then removed. A series of tested compound samples were added inside the wells arranged into six series of concentrations. A series of tested compound samples, arranged into six series of concentrations, were added inside the wells. Each series of tested compounds were filled into 3 wells (triplo). Used as cell control, the other three wells were not added with test compounds. While the other three have DMSO 1\% solution added in culture media as solution control. The cells were stored in $\mathrm{CO}_{2}$ incubator for 24 hours. Reagent MTT $0,5 \mathrm{mg} / \mathrm{mL}$ was prepared by diluting $1 \mathrm{~mL}$ of MTT solution into PBS $(5 \mathrm{mg} / \mathrm{mL})$ with media culture up to $10 \mathrm{~mL}$. The plate was removed from the incubator, media was discarded. Using PBS, cells were rinsed. Each well was added with $100 \mu \mathrm{L}$ of MTT reagent, including the media control with no cells. Cells were then incubated for 3 hours in order for a blue formazan product to yield. A $100 \mu \mathrm{L}$ of SDS $10 \%$ in $0,1 \mathrm{~N} \mathrm{HCl}$. was filled into every well. The paper-wrapped plate was incubated in the dark, in-room temperature overnight, outside the incubator. Plate cover and wrap were then removed and cell absorbance in each well was detected using ELISA-reader on a wavelength of $\lambda=595$ nm. ${ }^{17}$

\section{QSAR Equation}

In order to obtain QSAR equation, a psycho-chemical parameter that is frequently used in a structureactivity relationship was applied. As for lipophilic parameters, Clog P (molecule properties) calculation was adopted using the ChemBioDraw Ultra computer program and $\pi$ (group properties) with values displayed on the literature table. The electronic parameter was determined by examining the value of $\sigma$ (group properties) from the table and Etot value (molecule properties) was derived from Chem Office program. Steric parameter was determined by checking on CMR value (molecule properties) through the ChemBioDraw Ultra computer program and $\mathrm{E}$ value $\mathrm{E}_{\mathrm{s}}$ Taft (group properties).

Using the data of psycho-chemical properties parameter and the result of cytotoxic activity, a correlation test was done using SPPS program to obtain Quantitative Structure - Cytotoxic Activity Relationship or QSAR Equation. The best QSAR equity outcome can be further employed to acquire novel derivative compounds of N-benzoyl-N'-phenylthiourea, which is predicted to be the candidate for a more potent drug for breast cancer. 


\section{RESULTS AND DISCUSSION}

New compounds in Fig.-1(a,b,d,e) are synthesized from benzoyl chloride derivatives with N-phenyl thiourea in one stage. The five compounds in Fig.-1(a-e) were yellow or white solids and insoluble substances in water.

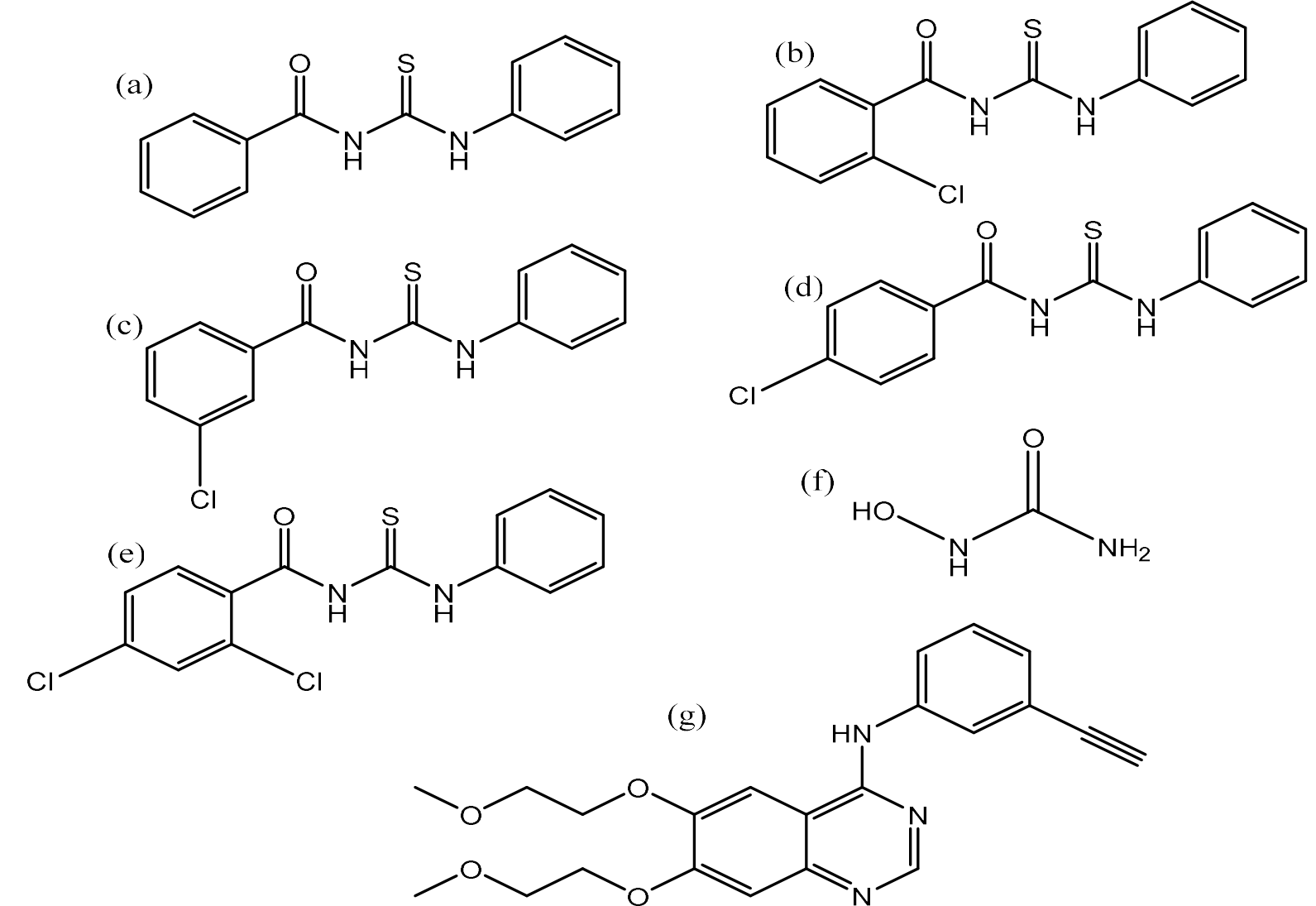

Fig.-1: Structure of (a) BFTU; (b) 2-Cl-BFTU; (c)3-Cl-BFTU; (d) 4-Cl-BFTU; (e)2,4-Cl-BFTU, (f)HU and (g)Erlotinib

The structure of the compound is synthesized and identified by IR spectroscopy, ${ }^{1} \mathrm{H}-\mathrm{NMR},{ }^{13} \mathrm{C}-\mathrm{NMR}$, and HRMS as follows:

\section{N-benzoyl-N'-phenylthiourea (BFTU)}

Yellow crystal, 71,78\%, m.p. $128^{\circ} \mathrm{C} .{ }^{1} \mathrm{H}-\mathrm{NMR}$ (DMS0-d6, $\left.400 \mathrm{MHz}\right): \delta 7.30(\mathrm{t}, \mathrm{J}=7.2 \mathrm{~Hz}, 1 \mathrm{H}, \mathrm{Ar}-\mathrm{H}) ; \delta$ $7.43(\mathrm{dd}, \mathrm{J}=7.2 ; 8.0 \mathrm{~Hz}, 2 \mathrm{H}, \mathrm{Ar}-\mathrm{H}) ; \delta 7.54(\mathrm{t}, \mathrm{J}=7.2 \mathrm{~Hz}, 2 \mathrm{H}, \mathrm{Ar}-\mathrm{H}) ;$ ); $\delta .65$ (dd, J=7.2;1.2 Hz, $1 \mathrm{H}, \mathrm{Ar}-\mathrm{H})$; ); $\delta .69(\mathrm{~d}, \mathrm{~J}=8.0 \mathrm{~Hz}, 2 \mathrm{H}, \mathrm{Ar}-\mathrm{H}) ; \delta 7.98(\mathrm{dd}, \mathrm{J}=7.2 ; 1.2 \mathrm{~Hz}, 2 \mathrm{H}, \mathrm{Ar}-\mathrm{H}) ; \delta 11.56(\mathrm{~s}, 1 \mathrm{H}, \mathrm{O}=\mathrm{C}-\mathrm{NH}-\mathrm{C}=\mathrm{S}) ; \delta$ $12.61\left(\mathrm{~s}, 1 \mathrm{H}, \mathrm{S}=\mathrm{C}-\mathrm{NH}-\mathrm{CH}_{2}\right)$. NMR ${ }^{13} \mathrm{C}(\mathrm{DMSO}-\mathrm{d} 6,100 \mathrm{MHz}) ; \delta 124.9$ (2C, Ar); $\delta 126.9$ (2C, Ar); $\delta$ 129.0(2C, Ar); $\delta 129.0$ (1C, Ar); $\delta 129.2$ (2C, Ar); $\delta 132.7$ (1C, Ar); $\delta 133.7$ (1C, Ar); $\delta 138.5$ (1C, Ar); $\delta$ $168.8(1 \mathrm{C}, \mathrm{C}=\mathrm{O}) ; \delta 179.7(1 \mathrm{C}, \mathrm{C}=\mathrm{S})$. IR $(\mathrm{KBr}), v$ maks $\left(\mathrm{cm}^{-1}\right): 1672(\mathrm{C}=\mathrm{O}$ amide $) ; 1606 \& 1488(\mathrm{C}=\mathrm{C} \mathrm{Ar})$; 3280\&1606 (NH strech sec.amides); 1083\&815 (C=S). HRMS (m/z) $\mathrm{C}_{14} \mathrm{H}_{11} \mathrm{~N}_{2} \mathrm{OS}:(\mathrm{M}-\mathrm{H})^{-}: 255.0590$, Calc. Mass: $255.0592 \delta \mathrm{m} / \mathrm{z}=0.0002<0.005$.

\section{N-(2-chloro)benzoyl-N'-phenylthiourea (2-Cl-BFTU)}

White crystal, 51,90\%, m.p. $120^{\circ} \mathrm{C} .{ }^{1} \mathrm{H}-\mathrm{NMR}$ (DMSO-d6, $\left.400 \mathrm{MHz}\right): \delta$ 7,20 (t, $\left.J=7,8 \mathrm{~Hz}, 1 \mathrm{H}, \mathrm{Ar}-\mathrm{H}\right) ; \delta$ $7,40$ (t, $J=7,8 \mathrm{~Hz}, 2 \mathrm{H}, \mathrm{Ar}-\mathrm{H}) ; \delta 7,48$ (dd, $J=8,0 ; 1,8 \mathrm{~Hz}, 2 \mathrm{H}, \mathrm{Ar}-\mathrm{H}) ; \delta 7,52$ (dd, $J=8,0 ; 1,8 \mathrm{~Hz}, 2 \mathrm{H}, \mathrm{Ar}-\mathrm{H}) ; \delta$ $7,70(\mathrm{~d}, J=7,8 \mathrm{~Hz}, 2 \mathrm{H}, \mathrm{Ar}-\mathrm{H}) ; \delta 11,96(\mathrm{~s}, 1 \mathrm{H}, \mathrm{O}=\mathrm{C}-\mathrm{NH}-\mathrm{C}=\mathrm{S}) ; \delta 12,32(\mathrm{~s}, 1 \mathrm{H}, \mathrm{S}=\mathrm{C}-\mathrm{NH}-\mathrm{Ar}) . \mathrm{NMR}{ }^{13} \mathrm{C}$ (DMSO-d6, $100 \mathrm{MHz}$ ) $\delta$ 127,0 (1C, Ar); $\delta 127,7$ (1C, Ar); $\delta 128,9$ (1C, Ar); $\delta 129,3$ (1C, Ar); $\delta 129,5$ (1C, Ar); $\delta 129,7$ (1C, Ar); $\delta 129,9$ (1C, Ar); $\delta 130,1$ (1C, Ar); $\delta 130,5$ (1C, Ar); $\delta 132,6(1 \mathrm{C}, \mathrm{Ar}) ; \delta 134,9$ $(1 \mathrm{C}, \mathrm{Ar}) ; \delta 138,4(1 \mathrm{C}, \mathrm{Ar}) ; \delta 168,3(1 \mathrm{C}, \mathrm{C}=\mathrm{O}) ; \delta 179,2(1 \mathrm{C}, \mathrm{C}=\mathrm{S}) . \mathrm{IR}(\mathrm{KBr}), v$ maks $\left(\mathrm{cm}^{-1}\right): 1681(\mathrm{C}=\mathrm{O}$ 
RASĀYAN J. Chem.

Vol. 14 | No. 4 |2698-2704| October- December | 2021

amide) ; 1681\&1499 (C=C Ar); 3157\&1594 (NH strech sec. amide); 1105\&832 (C=S), HRMS (m/z) $\mathrm{C}_{14} \mathrm{H}_{10} \mathrm{~N}_{2} \mathrm{OSCl}:(\mathrm{M}-\mathrm{H})^{-}=289,0203$, Calc.Mass $=289,0202 . \delta \mathrm{m} / \mathrm{z}=0,0001<0,005$.

\section{N-(3-chloro)benzoyl-N'-phenylthiourea (3-Cl-BFTU)}

Yellow crystal, 45,40\%, m.p. $119^{0} \mathrm{C} .{ }^{1} \mathrm{H}-\mathrm{NMR}$ (DMSO-d6, $\left.400 \mathrm{MHz}\right): \delta 7.24$ (t, J=7.2 Hz, $\left.1 \mathrm{H}, \mathrm{Ar}-\mathrm{H}\right) ; \delta$ $7.39(\mathrm{t}, \mathrm{J}=7.2 ; 8.0 \mathrm{~Hz}, 2 \mathrm{H}, \mathrm{Ar}-\mathrm{H}) ; \delta 7.52(\mathrm{t}, \mathrm{J}=8.0 \mathrm{~Hz}, 1 \mathrm{H}, \mathrm{Ar}-\mathrm{H}) ;) ; \delta 7.66(\mathrm{~d}, \mathrm{~J}=8.0 \mathrm{~Hz}, 1 \mathrm{H}, \mathrm{Ar}-\mathrm{H}) ;$ ); $\delta 7.67$ $(\mathrm{d}, \mathrm{J}=8.0 \mathrm{~Hz}, 2 \mathrm{H}, \mathrm{Ar}-\mathrm{H}) ; \delta 7.88$ (d, J=8.0 Hz, 1H, Ar-H); $\delta .99$ (s, 1H, Ar-H); $\delta 11.75$ (s, 1H,O=C-NH$\mathrm{C}=\mathrm{S}) ; \delta 12.46(\mathrm{~s}, 1 \mathrm{H}, \mathrm{S}=\mathrm{C}-\mathrm{NH}-\mathrm{Ar}) . \mathrm{NMR}{ }^{13} \mathrm{C}$ (DMSO-d6, $\left.100 \mathrm{MHz}\right) ; \delta 124.8(1 \mathrm{C}, \mathrm{Ar}) ; \delta 126.9(2 \mathrm{C}, \mathrm{Ar}) ;$ $\delta 128.0$ (1C, Ar); $\delta 129.0$ (1C, Ar); $\delta 129.2$ (2C, Ar); $\delta 130.9$ (1C, Ar); $\delta 133.3$ (1C, Ar); $\delta 133.7$ (1C, Ar); $\delta 134.8(1 \mathrm{C}, \mathrm{Ar}) ; \delta 138.5(1 \mathrm{C}, \mathrm{Ar}) ; \delta 167.4(1 \mathrm{C}, \mathrm{C}=\mathrm{O}) ; \delta 179.5(1 \mathrm{C}, \mathrm{C}=\mathrm{S}) . \mathrm{IR}(\mathrm{KBr}), v$ maks $\left(\mathrm{cm}^{-1}\right): 1672$ $(\mathrm{C}=\mathrm{O}$ amide $) ; 1672 \& 1451(\mathrm{C}=\mathrm{C}$ Ar); 3219\&1592 (NH strech sec.amides); 1085\&811 (C=S). HRMS (m/z) $\mathrm{C}_{14} \mathrm{H}_{10} \mathrm{~N}_{2} \mathrm{OSCl}:(\mathrm{M}-\mathrm{H})^{-}: 289.0200$, Calc. Mass : 289.0202. $\delta \mathrm{m} / \mathrm{z}=0.0002<0.005$.

\section{N-(4-chloro)benzoyl-N'-phenylthiourea (4-Cl-BFTU)}

Yellow crystal, 61,72\%, m.p. $126^{0} \mathrm{C} .{ }^{1} \mathrm{H}-\mathrm{NMR}$ (DMSO-d6, $\left.400 \mathrm{MHz}\right): \delta$ 7,24 (t, $J=7,2 \mathrm{~Hz}, 1 \mathrm{H}, \mathrm{Ar}-\mathrm{H}$ ); $\delta$ $7,39$ (t, $J=7,2 \mathrm{~Hz}, 2 \mathrm{H}, \mathrm{Ar}-\mathrm{H}) ; \delta 7,58(\mathrm{~d}, J=8,4,2 \mathrm{H}, \mathrm{Ar}-\mathrm{H}) ; \delta 7,65$ (d, $J=7,2 \mathrm{~Hz}, 2 \mathrm{H}, \mathrm{Ar}-\mathrm{H}) ; \delta 7,96(\mathrm{~d}, J=8,4$ $\mathrm{Hz}, 2 \mathrm{H}, \mathrm{Ar}-\mathrm{H}) ; \delta 11,63$ (s,1H, O=C-NH-C=S); $\delta 12,47$ (s,1H, S=C-NH-Ar). NMR ${ }^{13} \mathrm{C}$ (DMSO-d6, 100 $\mathrm{MHz}) ; \delta 124,9$ (2C, Ar); $\delta 126,9$ (2C, Ar); $\delta 126,9$ (1C, Ar); $\delta 129,1$ (2C, Ar); $\delta 129,2$ (2C, Ar); $\delta 131,2(1 \mathrm{C}$, $\mathrm{Ar}) ; \delta 131,6(1 \mathrm{C}, \mathrm{Ar}) ; \delta 138,5(1 \mathrm{C}, \mathrm{Ar}) ; \delta 167,8(1 \mathrm{C}, \mathrm{C}=\mathrm{O}) ; \delta 179,5(1 \mathrm{C}, \mathrm{C}=\mathrm{S}) . \mathrm{IR}(\mathrm{KBr}), v \mathrm{maks}\left(\mathrm{cm}^{-1}\right)$ : 1667 ( $\mathrm{C}=\mathrm{O}$ amide) ; 1667\&1482 ( $\mathrm{C}=\mathrm{C}$ Ar); 3333\&1593 (NH strech sec.amides); 1092\&831 (C=S). HRMS $(\mathrm{m} / \mathrm{z}) \mathrm{C}_{14} \mathrm{H}_{10} \mathrm{~N}_{2} \mathrm{OSCl}:(\mathrm{M}-\mathrm{H})^{-}=289,0204$, Calc. Mass $=289,0202 . \delta \mathrm{m} / \mathrm{z}=0,0002<0,005$.

\section{N-(2,4-dichloro)benzoyl-N'-phenylthiourea (2,4-2Cl-BFTU)}

White crystal, 33,81\%, m.p. $118^{0} \mathrm{C} .{ }^{1} \mathrm{H}-\mathrm{NMR}$ (DMSO-d6, $\left.500 \mathrm{MHz}\right): \delta 7,29$ (t, $J=7,8 \mathrm{~Hz}, 1 \mathrm{H}, \mathrm{Ar}-\mathrm{H}$ ); $\delta$ $7,38(\mathrm{dd}, J=8,3 ; 2.0 \mathrm{~Hz}, 1 \mathrm{H}, \mathrm{Ar}-\mathrm{H}) ; \delta 7,40(\mathrm{~d}, J=8,3 \mathrm{~Hz}, 1 \mathrm{H}, \mathrm{Ar}-\mathrm{H}) ; \delta 7,42(\mathrm{t}, J=7,8 \mathrm{~Hz}, 2 \mathrm{H}, \mathrm{Ar}-\mathrm{H}) ; \delta 7,50$ $(\mathrm{d}, J=2,0 \mathrm{~Hz}, 1 \mathrm{H}, \mathrm{Ar}-\mathrm{H}) ; \delta 7.68$ (d, $J=7,8 \mathrm{~Hz}, 2 \mathrm{H}, \mathrm{Ar}-\mathrm{H}) ; \delta 9,46(\mathrm{~s}, 1 \mathrm{H}, \mathrm{O}=\mathrm{C}-\mathrm{NH}-\mathrm{C}=\mathrm{S}) ; \delta 12,29$ (s, $1 \mathrm{H}, \mathrm{S}=\mathrm{C}-$ NH-Ar) . NMR ${ }^{13} \mathrm{C}$ (DMSO-d6, $\left.125 \mathrm{MHz}\right) ; \delta$ 124,2 (2C, Ar); $\delta$ 127,2 (1C, Ar); $\delta 128,1(1 \mathrm{C}, \mathrm{Ar}) ; \delta 129,1$ (2C, Ar); $\delta 130,6$ (1C, Ar); $\delta 130,9$ (1C, Ar); $\delta 131,6$ (1C, Ar); $\delta 132,3$ (1C, Ar); $\delta 137,4$ (1C, Ar); $\delta 139,2$ $(1 \mathrm{C}, \mathrm{Ar}) ; \delta 165,3(1 \mathrm{C}, \mathrm{C}=\mathrm{O}) ; \delta 177,7(1 \mathrm{C}, \mathrm{C}=\mathrm{S})$. IR $(\mathrm{KBr}), v$ maks $\left(\mathrm{cm}^{-1}\right): 1686(\mathrm{C}=\mathrm{O}$ amide $) ; 1686 \& 1471$ $\left(\mathrm{C}=\mathrm{C}\right.$ Ar); 3168\&1593 (NH strech sec.amides); 1098\&823 (C=S). HRMS (m/z) $\mathrm{C}_{14} \mathrm{H}_{9} \mathrm{~N}_{2} \mathrm{OSCl}_{2}:(\mathrm{M}-\mathrm{H})^{-}=$ 322,9816 , Calc. Mass $=322,9813 . \delta \mathrm{m} / \mathrm{z}=0,0003<0,005$.

Table-1: RS, $\mathrm{IC}_{50}$ MCF-7 and Vero Cells Value of 5 Test Compounds and 2 Reference

\begin{tabular}{l|c|c}
\hline $\begin{array}{c}\text { Test and Reference } \\
\text { Compounds }\end{array}$ & $\begin{array}{c}\mathrm{IC}_{50} \mathrm{MCF}-7 \text { Cell Line } \\
(\mathrm{mM}) \pm \mathrm{SD}\end{array}$ & $\begin{array}{c}\mathrm{IC}_{50} \text { Vero Cell Line } \\
(\mathrm{mM}) \pm \mathrm{SD}\end{array}$ \\
\hline BFTU & $0.98 \pm 0.0145$ & $49.40 \pm 0.0023$ \\
\hline 2-Cl-BFTU & $0.37 \pm 0.0145$ & $24.55 \pm 0.0025$ \\
\hline 3-Cl-BFTU & $0.43 \pm 0.0242$ & $35.76 \pm 0.0017$ \\
\hline 4-Cl-BFTU & $0.53 \pm 0.0242$ & $76.10 \pm 0.0020$ \\
\hline 2,4-Cl-BFTU & $0.31 \pm 0.0130$ & $179.48 \pm 0.0030$ \\
\hline Hydroxyurea (HU) & $9.76 \pm 0.0183$ & $369.88 \pm 0.0025$ \\
\hline Erlotinib & $0.92 \pm 0.0215$ & $300.67 \pm 0.0015$ \\
\hline
\end{tabular}

Synthesized from N-phenylthiourea using an acylation process with benzoyl chloride and its derivatives, N-benzoyl-N'-phenylthiourea (BFTU) and its 4 derivatives were obtained. ${ }^{16}$ As shown in Table 1, Nbenzoyl-N'-phenylthiourea and its 4 derivatives demonstrate greater cytotoxic activities when compared with both hydroxyurea and erlotib cancer drugs. Moreover, this synthesized compound and its derivatives exhibit selectivity results, as much as 50 to 579 times more selective on breast cancer cell MCF-7. In conclusion, N-benzoyl-N'-phenylthiourea and its 4 derivatives are toxic to breast cancer cell MCF-7, yet they are either selective or non-toxic to Vero normal cells. In addition, the cytotoxic activity is also influenced by the steric and electronic effects of the added functional groups. This could be seen from the IC50 value of the 2,4-Cl BFTU compound with the most potent activity $(0.31 \mathrm{mM}) .{ }^{23}$ The addition of $\mathrm{Cl}$ groups at positions 2 and 4 can increase its activity where the electronic factor produced was greater and the bulk of the compound was also in accordance with the MCF-7 cell receptor. 


\section{RASĀYAN J. Chem.}

Vol. 14 | No. 4 |2698-2704| October- December | 2021

Table-2: Parameter Value of Psychochemical Property and Cytotoxic Activity of N-Benzoyl-N'-Phenylthiourea Compound and its 4 Derivatives

\begin{tabular}{c|l|l|c|c|c|c|c|c}
\hline No & Compounds & \multicolumn{6}{|c|}{ Psychochemical Properties } & Activity \\
\cline { 3 - 9 } & & Clog P & $\pi$ & $\sigma$ & Etot & Es Taft & CMR & Log 1/IC 50 \\
\hline 1. & BFTU & 2.5600 & 0.0000 & 0.0000 & -0.4727 & 1.2400 & 7.7624 & 0.0088 \\
\hline 2. & 2-Cl-BFTU & 2.6400 & 0.7100 & 0.2300 & -2.4661 & 0.2700 & 8.2838 & 0.4318 \\
\hline 3. & 3-Cl-BFTU & 3.4700 & 0.7600 & 0.3700 & -9.7426 & 0.2700 & 8.2838 & 0.3665 \\
\hline 4. & 4-Cl-BFTU & 3.4700 & 0.7000 & 0.2300 & -1.2393 & 0.2700 & 8.2838 & 0.2757 \\
\hline 5. & 2,4-2Cl-BFTU & 3.4300 & 1.4200 & 0.4600 & -10.4193 & 0.5400 & 8.7752 & 0.5086 \\
\hline
\end{tabular}

\section{QSAR Equation Results}

1. $\log 1 / \mathrm{IC}_{50}=0.203 \mathrm{Clog} \mathrm{P}-0,314$

$(n=5 ; r=0.495 ; \mathrm{SE}=0.194 ; \mathrm{F}=0.974 ; \mathrm{Sig}=0.396)$

2. $\log 1 / \mathrm{IC}_{50}=0,033 \mathrm{Clog}^{2}-0,008$

$(\mathrm{n}=5 ; \mathrm{r}=0.487 ; \mathrm{SE}=0.195 ; \mathrm{F}=0.931 ; \mathrm{Sig}=0.406)$

3. $\log 1 / \mathrm{IC}_{50}=0.354 \pi+0.064$

$(\mathrm{n}=5 ; \mathrm{r}=0.922 ; \mathrm{SE}=0.864 ; \mathrm{F}=16.953 ; \mathrm{Sig}=0.026)$

4. $\log 1 / \mathrm{IC}_{50}=0.193 \pi^{2}+0.180$

$(\mathrm{n}=5 ; \mathrm{r}=0.792 ; \mathrm{SE}=0.144 ; \mathrm{F}=4.155 ; \mathrm{Sig}=0.134)$

5. $\log 1 / \mathrm{IC}_{50}=-0,027$ Etot +185

$$
(\mathrm{n}=5 ; \mathrm{r}=0.681 ; \mathrm{SE}=0.163 ; \mathrm{F}=2.597 ; \mathrm{Sig}=0.205)
$$

6. $\log 1 / \mathrm{IC}_{50}=1.002 \sigma+0.060$

$$
(\mathrm{n}=5 ; \mathrm{r}=0.905 ; \mathrm{SE}=0.095 ; \mathrm{F}=13.500 ; \mathrm{Sig}=0.035)
$$

7. $\log 1 / \mathrm{IC}_{50}=0,497 \mathrm{CMR}-3,793$

$(\mathrm{n}=5 ; \mathrm{r}=0.822 ; \mathrm{SE}=0.086 ; \mathrm{F}=16.940 ; \mathrm{Sig}=0.026)$

8. $\log 1 / \mathrm{IC}_{50}=-0.352 \mathrm{Es}+0.501$

$(\mathrm{n}=5 ; \mathrm{r}=0.767 ; \mathrm{SE}=0.143 ; \mathrm{F}=4.284 ; \mathrm{Sig}=0.130)$

\section{The Best QSAR Equation, namely}

$\log 1 / \mathrm{IC}_{50}=0.354 \pi+0.064(\mathrm{n}=5 ; \mathrm{r}=0.922 ; \mathrm{SE}=0.864 ; \mathrm{F}=16.953 ; \mathrm{Sig}=0.026)$

Equation No. 3 was selected due to its significance value of $0,026(\alpha<0.05)$ with correlation coefficient of $\mathrm{r}=0.922, \mathrm{~F}=16.953$ dan $\mathrm{SE}=0.864$. This was the greatest value among other equations. The selected equation explains that lypophilic properties using a group approach greatly influence the cytotoxic activity on MCF-7 cells of N-benzoyl-N'-phenylthiourea and its derivatives. ${ }^{24}$

After acquiring the foremost correlation of QSAR equation as mentioned above, it is crucial to note that it is the substituent with a strong lipophilic property $(\pi)$ that affects cytotoxic activity rather than electronic $(\sigma)$ or steric property (Es). This QSAR equation will minimize trials and errors in the effort of advancing the derivatives of N-benzoyl-N'-phenylthiourea as cancer drugs in the future.

\section{CONCLUSION}

From result and discussion section above, several novel findings from this study are reported that The best Quatitative Structure - Cytotoxic Activity Relationship or QSAR Equation was obtained from N-benzoylN'-phenylthiourea compound and its 4 derivatives, namely:

$\log 1 / \mathrm{IC}_{50}=0.354 \pi+0.064$

$(\mathrm{n}=5 ; \mathrm{r}=0.922 ; \mathrm{SE}=0.864 ; \mathrm{F}=16.953 ; \operatorname{Sig}=0.026)$

\section{ACKNOWLEDGEMENT}

This study was supported by the General Directorate of Resources for Science, Technology and Higher Education of Ministry of Research, Technology and Higher Education (KEMRISTEK DIKTI) with the scheme of scholarship funding for the Ph.D. program at Airlangga University, Surabaya, Indonesia.

\section{REFERENCES}

1. Siswandono, Development of New Drugs, Airlangga University Press, Surabaya, (2014)

2. D. Abraham, Burger's Medicinal Chemistry and Drug Discovery, $6^{\text {th }} \mathrm{ed}$, Volume 1: Drug Discovery, John Wiley and Sons, New York, (2003) 
RASĀYAN J. Chem.

Vol. 14 | No. 4 |2698-2704| October- December | 2021

3. Ministry of Health RI. Infodatin: Stop Cancer, Center for Data and Information Ministry of Health, (online, 2015), accessible on 24-08-2019.

4. A. Tartarone, C. Lazzari, R. Lerose, V. Conteduca, G. Improta,; A. Zupa, A. Bulotta, M. Aieta, V. Gregorc, Lung Cancer, 81, 3, 328-336 (2013), https://doi.org/10.1016/j.lungcan.2013.05.020

5. D. Kesuma,; Siswandono; B.T. Purwanto,; M. Rudyanto, Journal of Chinese Pharmaceutical Science, 27(10), 696(2018), https://doi.org/10.5246/jcps.2018.10.071

6. A. Shakeel, A. A.Altaf, A. M.Qureshi, A. Badshah, Journal of Drug Design and Medicinal Chemistry, 2(1),10-20 (2016), https://doi.org/10.11648/J. JDDMC.20160201.12

7. H.Q. Li, Y. Yan, L. Shi, C.F. Zhou, H. Zhu, Bioorganic Medicinal Chemistry, 18, 305(2010), https://doi.org/10.1016/i.bmc.2009.10.054

8. L.I.J ian, T. Jin-zhi,; C. Li-li,; Z. Jiang, , S. Xu, M. Chang-lin, F. Li-li,; L. Li-pig, D. Jian,, X. Xishan,; L. Hong, L. Xiao-min, J. Huan-liang, Design, Acta Pharmacologica, 27(9), 1259( 2006), https://doi.org/10.1111/j.1745-7254.2006.00437.x

9. D. Kesuma, Siswandono, B.T. Purwanto, M. Rudyanto, Journal of Chinese Pharmaceutical Science, 29(2),123(2020), https://doi.org/10.5246/jcps.2020.02.010

10. E. Dhineshkumar, M. Iyappan and C. Anbuselvan, Rasayan Journal of Chemistry, 13(4), 2405(2020), https://doi.org/10.31788/RJC.2020.1346041

11. J.G. Topliss, Journal of Medicinal Chemistry, 15(10),1006(1972), https://doi.org/10.1021/jm00280a002

12. J.M. Beale, J.H. Block, Wilson and Gisvolds Textbook of Organic Medicinal and Pharmaceutical Chemistry, $12^{\text {th }}$ ed, Lippincott Williams \& Wilkins, Philadelphia, (2011)

13. Y.C.Martin, Quantitative of Drug Design, Critical Introduction. $2^{\text {nd }}$ ed, CRC Press, Boca Raton, (2010)

14. J. Clayden, N. Greeves, S. Warren, P.Wothers, Organic Chemistry, $2^{\text {nd }}$ ed. Oxford University Press, New York, 279-289 (2012)

15. D.L. Pavia, G.M. Lampman, G.S.Kriz, R. James, J.R. Vyvyan, Spectroscopy, $4^{\text {th }}$ ed., Brooks/Cole, Belmont, pp.851-886 (2009)

16. D. Kesuma, A.L. Nasyanka, M. Rudyanto, S. Siswandono, B.T. Purwanto, and I.G.A Sumartha, Rasayan Journal of Chemistry, 13(3),1914(2020), https://doi.org/10.31788/RJC.2020.1335694

17. Cancer Chemoprevention Research Center Faculty of Pharmacy UGM (CCRC-UGM), Fixed procedure Cytotoxic Test Method MTT, (2012)

18. F.A. Shepherd, J.R. Pereira,; T. Ciuleanu, New England Journal of Medicine, 353, 123(2005), https://doi.org/10.1056/NEJMoa050753

19. A. Koc, L.J. Wheeler, C.K. Mathews, G.F. Merrill, Journal of Biological Chemistry, 279(1), 223(2004)

20. C. Avendaño and J.C. Menéndez, Medicinal Chemistry of Anticancer Drugs, $2^{\text {nd }}$ ed, Elsevier, Amsterdam, 15-19, 396-406 (2015)

21. W. Yang, Y. Hu, Y.S Yang, F. Zhang, Y.B. Zhang, X.L. Wang, J.F.Tang, W.Q Zhong, and H.L. Zhu, Bioorganic \& Medicinal Chemistry, 21, 1050(2013), https://doi.org/10.1016/j.bmc.2013.01.013

22. Siswandono, Medicinal Chemistry, 1, $2^{\text {nd }}$ ed, Airlangga University Press, Surabaya , pp.11-22, pp..360-381, pp.447-451 (2016)

23. Siswandono , R. Widyowati , A. Suryadi, T. Widiandani, and D. Prismawan, Rasayan Journal of Chemistry, 13(4), 2559(2020), https://doi.org/10.31788/RJC.2020.1345749

24. B. T. Purwanto, S. Hardjono , T. Widiandani, A. L. Nasyanka, I. Siswanto, Journal of Hunan University (Natural Sciences), 48(3), 78(2021)

[RJC-6357/2021] 